\title{
DISPUTAS DE TIERRAS Y AGUAS EN LA PLANICIE DE INUNDACIÓN DEL LAGO TONLÉ SAP DE CAMBOYA ${ }^{1}$
}

\author{
JOHN MARSTON \\ El Colegio de México \\ CHHUON HOEUR \\ Investigador independiente
}

El presente trabajo aborda la movilización de una comunidad y el conflicto suscitado en torno de los embalses de irrigación en la planicie de inundación del lago Tonlé Sap, en un subdistrito de la provincia de Kampong Thom de Camboya. La organización cooperativa que describiremos podría concordar con los modelos de Elinor Ostrom relativos a los recursos de uso común, ${ }^{2} \mathrm{y}$ a primera vista parece representar un proceso de empoderamiento, arraigado alrededor de los recursos. Sin embargo, en este ensayo, más que investigar las implicaciones del modelo de Ostrom, nos interesa desvelar y documentar los intrincados y moralmente complejos procesos de negociación sobre la tenencia de la tierra, así como las consideraciones ambientales, económicas, sociales y políticas de dichos procesos, los cuales consideramos que revelan, en buena medida, los cambios que están teniendo lugar en Camboya desde principios de la década de 1990.

Este artículo fue recibido por la dirección de la revista el 19 de febrero de 2014 y aceptado para su publicación el 24 de abril de 2014.

${ }^{1}$ El trabajo principal para este ensayo se llevó a cabo durante un periodo de investigación de cuatro meses, en 2010, y contó con el apoyo del Center for Khmer Studies, con fondos del Committee for American Overseas Research Centers. Mi agradecimiento al Centro de Estudios de Asia y África de El Colegio de México por su constante apoyo y a Wendy Medina por su trabajo en la adaptación de las referencias bibliográficas al sistema utilizado por esta revista.

${ }^{2}$ Elinor Ostrom, Governing the Commons: The Evolution of Institutions for Collective Action, Cambridge-Nueva York, Cambridge University Press, 1990. 
Aunque el artículo no pretende elaborar un modelo teórico unificado acerca de estos sucesos, es claro que tienen relación con las discusiones teóricas sobre el desarrollo de la "territorialización" ${ }^{3}$ en los procesos históricos y de la resistencia a esta territorialización. Esto abarca obras, como la de David Harvey, basadas en el objetivo de abordar la idea marxista de la acumulación primitiva, la cual reformula como la "acumulación por desposesión". ${ }^{4}$ Maurice Bloch, refiriéndose al marxismo, afirma: "toda su obra [de Marx y Engels] prueba que la propiedad es representada por la ideología como una relación entre la gente y las cosas, pero que en términos materiales se trata de una relación social". ${ }^{5}$ Nos interesan sobremanera las relaciones sociales cambiantes, vinculadas a las variaciones de regímenes de la tenencia de la tierra, pero reconocemos que la situación se complica por el hecho de que la tenencia de la tierra tiene implicaciones ecológicas, y que en tal contexto se introduce la relación entre la gente y "las cosas" (el ambiente natural). Más allá de todo esto - regresando, en cierto sentido, a Ostrom-, nos interesa la naturaleza de la comunidad en la sociedad camboyana ${ }^{6}$ (el tema de las circunstancias en las cuales puede ser movilizada una comunidad rural y hasta qué punto puede sostenerse una movilización), así como los escollos socioculturales y políticos con que se encuentra una movilización en el momento histórico presente.

Kampong Thom es una provincia predominantemente agrícola que forma parte de aquellas que bordean el gran lago Tonlé Sap, un punto geográfico clave de Camboya donde se localizan $32.75 \%$ de las parcelas agrícolas del país. Tal provincia

${ }^{3}$ Robert David Sack, Human Territoriality: Its Theory and History, Cambridge, Cambridge University Press, 1986; Peter Vandergeest y Nancy Lee Peluso, "Territorialization and State Power in Thailand", Theory and Society, vol. 24, núm. 3, 1995, pp. 385-426; Kim Sean Somatra, "Territoriality and State Power in Cambodia: The Case of Demarcation in Tonle Sap", tesis de doctorado, Universidad de Sidney, Sidney, 2013. 2003.

${ }^{4}$ David Harvey, The New Imperialism, Nueva York, Oxford University Press,

${ }^{5}$ Maurice Bloch, "La propiedad y el final de la alianza”, en M. Bloch (comp.), Análisis marxistas y antropología social, Barcelona, Anagrama, 1977, p. 242.

${ }^{6}$ John A. Marston, "Introducción", en John A. Marston (coord.), Antropología y comunidad en Camboya: Reflexiones sobre la obra de May Ebihara, México, El Colegio de México, 2011. 
posee $4.6 \%$ del total de la población del país (estimada en 14.6 millones de habitantes) y $14.5 \%$ de la población de las provincias que circundan el lago Tonlé Sap.? Su carácter rural se evidencia por el hecho de que su densidad de población es de 46 personas por kilómetro cuadrado, frente a las 64 personas por kilómetro cuadrado de todas las provincias que bordean el lago Tonlé Sap, y las 75 personas por kilómetro cuadrado del país. En conjunto, $80 \%$ de la población de Camboya vive en áreas rurales, y $72.3 \%$ de la población se dedica a la agricultura. De este modo, la zona objeto estudio es un área típica del país. ${ }^{8}$

Cualquier referencia a las tasas de pobreza debe tener en cuenta la extrema pobreza que existía en el país tras el periodo de Pol Pot, de 1975 a 1979; tasas que han descendido drásticamente. De acuerdo con una estimación, las tasas de pobreza se redujeron 12 puntos porcentuales de 1993 a 2004. ${ }^{9}$ No obstante, alrededor de $53.5 \%$ de la población de la provincia de Kampong Thom aún vive por debajo del nivel de pobreza, frente a 33\% de la población en la región del Tonlé Sap y 28\% de la población del país. Aunque no contamos con estadísticas específicas sobre el distrito de Stoung o del subdistrito de Samprouch, la observación general y nuestros estudios realizados en una aldea indican claramente que, a medida que nos alejamos de la capital del distrito por la carretera nacional en dirección al Tonlé Sap, gran parte de la población vive cerca del nivel de subsistencia, en casas sencillas de madera o de paja, construidas de manera tradicional sobre pilotes. La electricidad aún no llega a estas áreas, pero la población tiene acceso a la radio, e incluso a la televisión, mediante baterías recargables. Entre la población existen niveles significativos de endeudamiento, de modo que durante cierto tiempo, mientras realizábamos nuestra investigación, las instituciones que otorgan microcréditos negaron los préstamos al subdistrito de Samprouch a causa del elevado incumplimiento de los pagos.

7 National Institute of Statistics, General Population Census of Cambodia 2008: Provisional Population Totals, Phnom Penh, National Institute of Statistics, 2008.

${ }^{8}$ Department of Planning and Statistics, Agriculture Census of Cambodia, Phnom Penh, Ministry of Agriculture, Forestry and Fisheries, 18-21 de febrero de 2014.

9 Banco Mundial, Cambodia: Halving Poverty by 2014? Poverty Assessment 2006, Informe núm. 35213-KH, Nueva York, Banco Mundial. 
El subdistrito de Samprouch depende del distrito de Stoung, en la provincia de Kampong Thom, que se extiende desde la carretera nacional en dirección hacia el lago Tonlé Sap. El sistema ecológico del río y del lago Tonlé Sap tiene gran influencia en lo que describiremos en este trabajo. A medida que la tierra asciende suavemente desde la cuenca del lago, se observa el cambio de manglares a tierra de matorrales, luego a pastizales $y$, posteriormente, a los tipos de terrenos de arrozales inundados que constituyen un icono del paisaje de las tierras bajas de Camboya. Durante la época del año en que las aguas ascienden al máximo, las tierras se inundan casi hasta la carretera nacional. Este hecho en sí hace que las cuestiones sobre la tenencia de la tierra se vuelvan complicadas. ¿Quién tiene derecho a la tierra cuando no se encuentra inundada y a usar el agua que está retrocediendo después de la inundación para irrigar?

Tal como nos contó un funcionario de una organización no gubernamental, las áreas de manglares, pastizales y arrozales pueden cambiar con facilidad de una categoría a otra. Los manglares pueden desaparecer y convertirse en pastizales o en arrozales irrigados. Una vez abandonados, los arrozales vuelven a convertirse en pastizales y, posteriormente, en manglares. El sistema ecológico de esta región es especialmente delicado, y los manglares - que han disminuido en forma drástica en años recientes- son esenciales para el desove de los peces del Tonlé Sap ${ }^{10}$ y, de hecho, para la preservación del lago como un ecosistema. Asimismo, los pastizales constituyen refugios de especies de aves en peligro de extinción. ${ }^{11}$ Aparte de la agricultura del arroz, para la economía de la planicie de inundación también son importantes el pastoreo de ganado y la recolección de juncos y de otros productos no madereros. Además, son importantes las charcas y los lagos de la planicie de inundación para el cultivo de raíces comestibles de loto y, esencialmente, para la pesca.

${ }^{10}$ Mak Sithirith, Cooperation in the Mekong River Basin: A Reflection of Cambodia's Experiences in the Development of the Mekong Region, Chiang Mai, Regional Center for Social Science and Sustainable Development, 2007.

${ }^{11}$ N. E. Thomas Gray, Nigel J. Collar, Peter J. A. Davidson et al., "Distribution, Status, and Conservation of the Bengal Florican Houbaropsis Bengalensis in Cambodia", Bird Conservation International, vol. 19, núm. 1, 2009, pp. 1-14. 
Las carreteras nacionales que circundan el lago Tonlé Sap definen, en líneas generales, la extensión total de la planicie de inundación. El Ministerio del Medio Ambiente divide esta área, localizada dentro del círculo de las carreteras nacionales, en tres zonas: la zona más cercana a la carretera nacional, donde se permiten las tareas agrícolas y de desarrollo en forma libre; la zona más cercana al lago Tonlé Sap (en particular, el área de bosques de manglares), donde se prohíbe claramente cualquier actividad, y una zona de protección, localizada entre estas dos zonas, donde cualquier plan de desarrollo debe ser estudiado y negociado previamente.

Los aldeanos recuerdan que, en el pasado, los bosques de manglares llegaban muy cerca de la carretera nacional. (Al parecer, en la década de 1960 también se expandieron las áreas de desarrollo en la planicie de inundación. Resulta difícil saber qué tanto se extendía el área boscosa que recuerda la gente: si llegaba más allá de lo que abarcaba en la época de la preguerra que tuvo lugar durante el periodo de Pol Pot, y qué tanto se extendía durante la década de 1980 , cuando las preocupaciones por la seguridad motivaban a las poblaciones rurales a permanecer cerca de la carretera nacional.) A pesar de que el Tonlé Sap se localiza en el corazón mismo de las provincias dedicadas a la agricultura del arroz, las áreas más cercanas al lago tienen la reputación de ser "agrestes", allende el reino de la civilización normal. Además, en la década de 1980, durante los meses en que el agua tenía un nivel bajo, las guerrillas del Khmer Rouge usaban los manglares como sus bases.

Históricamente, la planicie de inundación del Tonlé Sap está vinculada al "arroz flotante", variedad de arroz de tallo largo que crece con la subida de las aguas procedentes de la inundación. ${ }^{12}$ Ta-Kouon, visitante chino del siglo XIII que viajó a la corte de Angkor, al parecer describe esta técnica, la cual aún se practicaba ampliamente en el periodo anterior al régimen de Pol Pot. Fox y Ledgerwood también han mostrado que era frecuente el uso históricamente significativo de irrigar el arroz con las aguas que se retiraban de la inundación; ${ }^{13}$ técnica

\footnotetext{
${ }^{12}$ Richard P. Lando y Solieng Mak, Deepwater Rice in Cambodia: A Baseline Survey, Manila, International Rice Research Institute, 1994.

${ }^{13}$ Jeff Fox y Judy Ledgerwood, "Dry-Season Flood-Recession Rice in the Me-
} 
que, a diferencia del arroz flotante (que crece con la subida del agua), consiste en capturar el agua cuando se retira de la planicie, después de la estación de las inundaciones, con el fin de utilizarla para irrigar.

En la década de 1980, tras el régimen de Pol Pot, el gobierno de la República Popular de Kampuchea trató de reintroducir el arroz flotante, y en la planicie de inundación se otorgaron lugares a ciertas aldeas y "grupos de solidaridad" con este propósito; posteriormente, cuando el sistema de "grupos de solidaridad" se desintegró, los lugares se asignaron a familias específicas. ${ }^{14}$ Sin embargo, el cultivo del arroz flotante nunca volvió a generalizarse. Se dan diversas razones para su fracaso: las cosechas no rendían frutos posiblemente, como mencionan algunos agricultores, debido a que ciertas variedades se habían perdido bajo el régimen del Khmer Rouge cuando los campesinos hambrientos se comían las semillas del arroz. También se ha dicho que los cambios ecológicos acaecidos en el lago han dado como resultado mayores variaciones en los niveles del agua, lo que hace que las cosechas de arroz flotante sean inestables. En la década de 1980, los habitantes de las aldeas también se negaban a cultivar en áreas alejadas de la carretera nacional por razones de seguridad: problemas de bandidaje y de guerrillas. Un informe del Centro de Estudios Avanzados también menciona los "desastres naturales (sequías, riadas, plagas y ganado deambulando)" como factores que influyeron para que se abandonara la planicie de inundación. ${ }^{15}$

Como consecuencia, tales intentos de reiniciar la agricultura del arroz flotante fueron en gran medida abandonados (aunque actualmente son causa de ciertas demandas por derechos

kong Delta: Two Thousand Years of Sustainable Agriculture?”, Asian Perspectives, vol. 38, núm. 1, 1999, pp. 37-50.

${ }^{14}$ Center for Advanced Study, "Justice for the Poor? An Exploratory Study of Collective Grievances and Land and Local Governance in Cambodia”, Phnom Penh, Center for Advanced Study-Banco Mundial, 2006.

${ }^{15}$ Ibid., p. 50. Sin embargo, tal como nos dijo un agricultor, puede haber habido otro factor, esto es, que en la década de 1980 no había comerciantes que compraran arroz en la estación seca. La intensificación de la agricultura seca del arroz que existe actualmente se da en conjunción con los comerciantes que lo compran, por lo que resulta explicable que a mediados de los ochenta descendiera este tipo de agricultura. 
de tierra). A nosotros se nos condujo a lugares aislados de la planicie de inundación del Tonlé Sap, situados en el distrito norte de Batheay, en la provincia de Kampong Cham, donde aún existe la agricultura del arroz flotante. No obstante, la impresión general de la mayor parte de los aldeanos camboyanos $\mathrm{y}$ de los funcionarios del gobierno con los que hablamos es que dicha práctica ha sido abandonada por completo.

Desde finales de la década de 1980 algunas instituciones internacionales han financiado diversos proyectos de irrigación a gran escala en el valle del río Mekong en su curso por Camboya, que incluye la cuenca del Tonlé Sap. Muchos de estos proyectos son expansiones o renovaciones de los sistemas de riego construidos originalmente antes de la guerra o durante el periodo de Pol Pot. En parte, a causa de su financiamiento internacional se ha escrito copiosamente sobre estos proyectos. ${ }^{16} \mathrm{La}$ mayor parte de dichos proyectos han consistido en irrigación por desviación de cauces, donde se utiliza el agua proveniente de los ríos y de los arroyos para destinarla a la irrigación. Con el apoyo de patrocinadores internacionales, el Ministerio de Recursos Hídricos y Mineralogía ha fomentado la formación de Grupos Campesinos de Usuarios de Agua, los cuales son objeto de algunos estudios. El aspecto general de esta política consiste en que los usuarios se coordinen mejor para usar el agua río arriba y río abajo. Muchos de los especialistas que han estudiado estos sistemas han estado afiliados en algún momento al Instituto Camboyano de Recursos para el Desarrollo.

${ }^{16}$ Véase Cambodian Development Resource Institute, Empirical Evidence of Irrigation Management in the Tonle Sap Basin: Issues and Challenges, documento de trabajo núm. 48, Phnom Penh, CDRI, 2010; Satu Muukkonen, "Water Management in Cambodia-Resources and Relations", tesis de maestría, Universidad de Helsinki, Helsinki, 2007; Joakim Öjendal, "Sharing the Good: Modes of Managing Water Resources in the Lower Mekong River Basin", tesis de doctorado, Universidad de Gotemburgo, Gotemburgo, 2000; Chea Chou, The Local Governance of Commonpool Resources: The Case of Irrigation Water in Cambodia, Phnom Penh, Cambodia Development Resource Institute, 2010; Daravy Khiev, "Challenge of Participant Irrigation Management in Cambodia: The Case of Damnak Ampil Irrigation Scheme”, tesis de maestría, Universidad de Sidney, Sidney, 2010; Bandeth Ros, "Participatory Irrigation Management and Factors that Influence the Success of Farmer-Water User Communities: A Case Study in Cambodia", tesis de maestría, Universidad de Massey, Auckland, 2010; Phalla Chem et al., Framing Research on Water Resources Management and Governance in Cambodia: A Literature Revierw, Phnom Penh, Cambodia Development Resource Institute, 2008. 
Ha habido escasas investigaciones sobre los sistemas de distribución del agua por gravedad, en los cuales se captan las aguas de las inundaciones en áreas cerradas para, posteriormente, cuando las aguas hayan retrocedido, utilizar gradualmente esta reserva para la irrigación; esto es, investigaciones sobre las diversas técnicas utilizadas durante el retiro de la inundación, aspectos que estudiaron Fox y Ledgerwood. En una escala reducida, dichos sistemas también implicaban históricamente la coordinación de la comunidad, que Middleton y Prom han descrito incluso como tradiciones localizadas de administración de recursos naturales basada en la comunidad. ${ }^{17}$ Algunos proyectos de irrigación a gran escala construidos durante el periodo de Pol Pot se valían de estas técnicas, entre ellos, dos proyectos en el distrito de Batheay, en Kampong Cham, que se han renovado satisfactoriamente desde la década de 1990 y que se han incorporado por completo a los esquemas del Ministerio de Recursos Hídricos y Mineralogía. ${ }^{18}$

El fenómeno que nos proporciona el marco para este trabajo lo constituye un esfuerzo, bastante repentino, para la construcción de esta clase de embalses en la planicie de inundación del Tonlé Sap durante la última década. Dicho esfuerzo lo emprendieron principalmente algunos empresarios, aunque también hubo una pequeña participación de aldeas, del gobierno subdistrital y, en forma cooperativa, de grupos de campesinos. Tales embalses irrigan las cosechas de la temporada seca donde se usan nuevas variedades de arroz de crecimiento rápido. El incremento de esta clase de embalses - los cuales, como veremos más adelante, han resultado ser muy controvertidos- ha tenido lugar predominantemente en las provincias de Bantheay Mean Chey, de Battambang, de Siem Reap y, sobre todo, de Kampong Thom, donde el gobernador provincial los ha promovido activamente. Los proyectos de irrigación por

${ }^{17}$ Carl Middleton y Tola Prom, "Community Organization for Managing Water Resources around Tonle Sap Lake: A Myth or a Reality”, en Matti Kummu, Marko Keskinen y Olli Varis (comps.), Modern Myths of the Mekong, Helsinki, Universidad Tecnológica de Helsinki, 2008, pp. 149-159.

${ }^{18}$ P. Someth, N. Kubo, H. Tanji y S. Ly, "Ring Dike System to Harness Floodwater from the Mekong River for Paddy Rice Cultivation in the Tonle Sap Lake Floodplain in Cambodia”, Agricultural Water Management, vol. 96, núm. 1, 2009, pp. 100-110. 
desvío de los cauces han sido más favorecidos en las provincias localizadas al suroeste del lago, en Kampong Chhnang y en Pursat; proyectos sobre los que se ha escrito más abundantemente. Aunque los embalses de irrigación empresariales han sido menos investigados que los proyectos de irrigación que han recibido patrocinio internacional, hay estudios notables hechos por el agrónomo Jean-Christophe Diepart y los especialistas en desarrollo Ngo Sothath y Chan Sophal. ${ }^{19}$ La cuestión más amplia que planteamos en nuestra investigación se refiere a la relación que existe entre estos proyectos de irrigación, que son básicamente empresariales, y las poblaciones rurales dedicadas a la agricultura, así como a los costos sociales y ecológicos de dichos proyectos en la primera década del siglo Xxi; aunque, para los propósitos de este trabajo, nos centraremos en un solo embalse de una cooperativa que se desarrolló como reacción a los embalses empresariales.

El florecimiento de los embalses de irrigación tiene dimensiones ambientales, sociales y políticas. Inicialmente hablaremos, en términos generales, de Kampong Thom, la provincia donde ha sido más intensa la construcción de embalses, y donde tal construcción ha causado más controversia; paulatinamente nos concentraremos más en los hechos del subdistrito de Samprouch, el lugar donde llevamos a cabo nuestro trabajo de campo.

Después de visitar la planicie de inundación durante la estación seca, y al ver las vastas extensiones de terreno, los defensores de los embalses piensan habitualmente en los grandes beneficios que obtendría Camboya si estas tierras, muchas de ellas lejos de los manglares, pudieran usarse en forma productiva.

${ }^{19}$ V'éase Jean-Christophe Diepart, "Problèmes et enjeux de l'économie rurale au Cambodge: Entre nouvelles gouvernances et réalités paysannes: Le cas de la province de Kampong Thom", tesis de doctorado, Académie Universitaire Wallonie-BruxellesFaculté Universitaire des Science Agronomiques de Gembloux, Communauté française de Belgique, 2007; Jean-Christophe Diepart, "Recent Land Dynamics in the Tonle Sap Flood Plain and its Impact on the Local Communities", Tonle Sap Biosphere Reserve Bulletin, vol. 3, 2007, pp. 20-22; Jean-Christophe Diepart, "Cambodian Peasant's Contribution to Rural Development: A Perspective from Kampong Thom Province”, Biotechnology, Agronomy, Society and Environment, vol. 14, núm. 2, 2010, pp. 321340; Ngo Sothath y Chan Sophal, Does Large Scale Agricultural Investment Benefit the Poor?, Phnom Penh, Cambodian Economic Association, junio de 2010. 
Las versiones sobre el inicio de la construcción de los embalses en el área varían, pero al parecer se debieron a los experimentos de agricultores y de hombres de negocios que habían participado en la agricultura del arroz flotante en Kampong Thom y en las áreas contiguas de la provincia de Siem Reap, y que buscaban alternativas prácticas. Esto captó el interés del gobernador provincial de Kampong Thom, Nam Tum, quien financió la construcción de embalses piloto a fin de depurar las técnicas, y posteriormente promovió cada vez más construcciones de estos embalses en la planicie de inundación. Un documento al que hemos tenido acceso data algunos de los embalses en los años 2001 y 2002, lo cual puede corresponder al tiempo en el que los empresarios comenzaron a demandar tierras. Los empresarios empezaron a construir con ímpetu en 2003, y en 2004 y 2005 las construcciones adquirieron gran impulso. En el subdistrito de Samprouch se inició una oleada de construcciones en 2004, cuando varios empresarios adquirieron terrenos para este propósito. Las cifras relativas a los embalses construidos varían, en parte porque no todas las listas incluyen los embalses más pequeños. Actualmente, hay alrededor de dieciocho en el subdistrito de Samprouch y más de 100 solamente en la provincia de Kampong Thom. Aunque la afectación social y ambiental fue mínima en un principio, esto comenzó a cambiar a medida que las redes de embalses a lo largo de la planicie de inundación eran cada vez más numerosas. Los campesinos del área inicialmente dudaban de que los embalses pudieran ser benéficos; no obstante, su actitud cambió cuando la tierra irrigada por los embalses se volvió sumamente productiva. Además, las grandes extensiones de terrenos eran muy adecuadas para las técnicas agrícolas mecanizadas.

Los terrenos que ocupa la planicie de inundación se consideran terrenos del Estado, y probablemente lo seguirán siendo en un futuro cercano, aunque parte del contexto de los sucesos que describimos corresponde al hecho de que está por introducirse el registro sistemático de la tierra a lo largo de todo el país. Esto significa que, entre otros aspectos, las poblaciones se encuentran particularmente preocupadas por el cambio de la tenencia de la tierra y ávidas de asegurarse un terreno con la esperanza de que pueda representar un acceso permanente a la tierra. 
La ley de 2001 relativa a la propiedad de la tierra introdujo la posibilidad de ofrecer concesiones de tierra con fines económicos. ${ }^{20}$ Tales concesiones ya han tenido repercusión en las localidades donde se han aplicado, $\mathrm{y}$ ha habido nutridas críticas de organizaciones no gubernamentales y de organismos internacionales por los efectos negativos para las poblaciones locales, donde ha existido la tradición comunal de acceso a la tierra. Por ejemplo, un informe de Derechos Humanos de las Naciones Unidas expresa sus:

[...] preocupaciones por no haber consultado con las comunidades locales, por la usurpación de la tierra y los efectos perjudiciales en los medios de subsistencia tradicionales, por los desplazamientos y los efectos adversos en el ambiente, por el empleo y las condiciones de trabajo, por la violencia y la intimidación y por la falta de una solución efectiva o de recursos para las comunidades afectadas. ${ }^{21}$

Aunque las mayores concesiones de tierras son autorizadas por la autoridad nacional, la ley originalmente incluía la disposición de otorgar concesiones de tierras más pequeñas, de hasta 100 hectáreas, autorizadas por las provincias. Sin embargo, en 2008 se cambió la ley para eliminar tales concesiones de tierras autorizadas por las provincias. La mayor parte de los embalses para irrigación en manos empresariales en Kampong Thom fueron autorizaciones provinciales, bajo los términos de esta disposición; aunque desde 2008 se han construido algunos embalses en tierras que se dice que fueron compradas. La mayor parte de estas tierras tienen un tamaño de entre 100 y 400 hectáreas, y vierten sus aguas en áreas de cultivo de arroz que son ligeramente mayores al tamaño del embalse.

De hecho, hay diversos grados de legalidad de los embalses de irrigación: los que se encuentran en tierras autorizadas subdistrital, distrital y provincialmente; los que únicamente están autorizados subdistrital y distritalmente sin nunca haber

${ }^{20}$ Kheang Un y Sokbunthoeun So, "Land Rights in Cambodia: How Neopatrimonial Politics Restricts Land Policy Reform”, Pacific Affairs, vol. 84, núm. 2, 2011, pp. 289-308.

${ }_{21}$ Cambodian Office of the High Commissioner for Human Rights, "Economic Land Concessions in Cambodia: A Human Rights Perspective", Phnom Penh, OHCHR, 2007, p. 14. 
contado con un permiso formal provincial; los que se construyeron sin ningún permiso, y los que se construyeron después de haber sido "comprados" a los campesinos (quienes pueden contar con derechos poco claros sobre la tierra). También está la cuestión de los embalses (o de las tierras de cultivo que dichos embalses alimentan) en relación con la ley ambiental. Y a ese respecto, el director de la oficina provincial del Ministerio de Agricultura, Silvicultura y Pesca, nos dijo que si los embalses violaban las zonas ambientales eran ilegales, incluso si habían sido aprobados por el gobierno provincial. Además, existen cuestiones relativas a si las tierras otorgadas por la provincia como concesiones de terrenos alguna vez se registraron como "tierras privadas del Estado", tal como se requiere para que sean reconocidas como concesiones de tierras. Por último, en Kampong Thom, los contratos para las concesiones de tierra estipulaban que la comunidad local debía explotar al menos $40 \%$ de la tierra en forma cooperativa si así lo requería dicha comunidad (aunque ha habido protestas por este requerimiento, tales acuerdos cooperativos se han dado en raras ocasiones, si acaso se han llegado a dar). Incluso en las mejores circunstancias, la legalidad incluye un elemento de negociación; esto ha sucedido en este caso, dado que las leyes son nuevas y ambiguas, y se relacionan con condiciones socioeconómicas que también se encuentran en un proceso de transformación.

Los agricultores generalmente se refieren a los grandes sistemas empresariales de irrigación como pertenecientes a las krumbum, o "compañías", lo que destaca hasta qué punto la gente reconoce que representan sistemas agrícolas cualitativamente diferentes a los de los propios campesinos; de hecho, hasta ahora los propietarios de los embalses han sido empresarios muy pequeños, con antecedentes en el arroz flotante, en cotos de pesca o como comerciantes. Algunos de ellos son personas de Siem Reap que proceden de familias antiguas, o con relaciones comerciales en el distrito; otros poseen negocios en la capital del distrito. Por ejemplo, uno de estos propietarios del subdistrito de Samprouch fue residente de este subdistrito durante mucho tiempo y su modesta casa se encuentra situada cerca de la carretera nacional. En muchos casos, los propietarios, más que caracterizarse por su riqueza, cuentan con ciertas 
conexiones que les permiten estar al tanto de las pautas del gobierno provincial; también cuentan con la posibilidad de obtener los préstamos disponibles. Desde la perspectiva de la mayor parte de los campesinos, los dueños de los embalses empresariales son de una riqueza que supera sus sueños. Hay ocasiones en que, vistos de esta manera, uno se ve tentado a considerarlos como los villanos del drama; sin embargo, no debe olvidarse que, para los estándares de Phnom Penh, su riqueza es modesta, y la inversión necesaria para construir un embalse de irrigación es más cercana a lo que se pagaría en Phnom Penh por una modesta tienda que forma parte de una casa al estilo chino, que lo que se necesitaría para construir un centro comercial. Para estos empresarios, las medidas del Estado destinadas a derribar los embalses representan un desastre. (Los defensores de los embalses dicen que los embalses están en la mira del Estado no por motivos ambientales, sino para dejar libres las tierras a inversionistas de mayor escala, que realmente son krumbum). ${ }^{22}$

Creemos que hasta ahora no existe documentación acerca del proceso por el cual los problemas ambientales planteados por los embalses atrajeron la atención pública e indujeron al primer ministro Hun Sen a pedir su demolición. A medida que los embalses se multiplicaban en la planicie de inundación, algunas organizaciones no gubernamentales, entre ellas el Equipo de Coalición en Defensa de la Pesca y la Sociedad para la Conservación de la Vida Silvestre, prestaban cada vez mayor atención al tema y recomendaban aplicar restricciones a la construcción de los nuevos embalses, por lo menos en algunas partes de la planicie de inundación, al tiempo que mantenían conversaciones con funcionarios del gobierno; no

${ }^{22}$ La evidencia de esta posibilidad es el hecho de que la Comisión de Desarrollo de Camboya cuenta con mapas que indican grandes superficies de la planicie de inundación como posibles lugares para que los inversionistas los exploten como concesiones de tierras. Algunas compañías chinas ya han hecho estudios de viabilidad de estas extensiones de tierras, estudios que, sin embargo, no han cuajado en proyectos concretos. La compañía Leopard Capital ha intentado concretar un gran proyecto en la planicie de inundación, el cual ya podría haber sido autorizado nacionalmente. Sin embargo, otra dimensión de toda esta situación es el hecho de que se ha encontrado petróleo en el lago Tonlé Sap y, en un momento dado, será explotado. Esto podría constituir otro factor para que el Estado restringiera el uso de las áreas de la planicie de inundación. 
obstante, probablemente la oposición a los embalses que tuvo mayor peso provino de ministerios del gobierno mismo. La oposición a los embalses se basaba principalmente en el temor de que la destrucción de los manglares provocara la desaparición de los peces del Tonlé Sap. Había otras preocupaciones en el sentido de que los embalses estuvieran situados de forma tan densa que interrumpieran los flujos de agua que se retiraba al lago. En ocasiones también bloqueaban los pasos tradicionales por donde los peatones, los ciclistas y los carros de bueyes accedían a las tierras bajas. Además, los agrónomos arguiían que la extraordinaria productividad inicial de la tierra sólo podría mantenerse en el futuro mediante el uso extensivo de fertilizantes, los cuales podrían tener un efecto negativo en el ecosistema y, en última instancia, poner en entredicho la rentabilidad de este sistema. En un discurso transmitido en repetidas ocasiones en la televisión, el primer ministro llegó a declarar que los embalses podrían provocar la desaparición del lago.

Todo esto dio como resultado que el gobierno nacional anunciara, en diciembre de 2009, que se debían destruir todos los embalses. Cuando el gobernador de Kampong Thom, Nam Tum, inicialmente aplazó las órdenes, se puso en marcha una lucha de poder entre él y Hun Sen; lucha que él no estaba en posibilidades de ganar y que tal vez endurecería la decisión de Hun Sen de eliminar los embalses. Cuando nos encontrábamos en Camboya examinando este asunto se destruyeron unos cuantos embalses dispersos durante la primera mitad de 2010; pero nos fue imposible determinar alguna pauta particular que guiara esa destrucción. Desde entonces ha habido informes de prensa sobre más embalses arrasados por motoniveladoras, un total de 45 en 2010, antes de que las lluvias hicieran imposible la tarea; aunque queda poco claro que todos vayan a ser destruidos. Un artículo del 9 de marzo, publicado en Phnom Penh Post, afirmaba que el proceso de demarcación que indicaría cuáles embalses debían ser destruidos tenía un avance de 25 por ciento. ${ }^{23}$

Todo esto proporciona el marco, como afirmamos al principio, para lo que constituye el tema principal de este trabajo; es-

${ }^{23}$ Sophakchakrya Khouth, "Progress Made on Illegal Reservoirs", Phnom Penh Post, 9 de marzo de 2011. 
to es, la movilización de poblaciones rurales relacionada con este fenómeno. Cualquier estudio adecuado de los embalses tiene que reconocer que éstos han sido el eje de conflictos significativos con las poblaciones locales. Los que apoyan los embalses empresariales sostienen generalmente que los campesinos eran indiferentes a éstos hasta que se hizo evidente que tales embalses eran muy rentables; sólo entonces decidieron que debían recibir una parte de sus beneficios. Es difícil evaluar dichos alegatos, dado que resulta complicado establecer una cronología de las protestas; sin embargo, a causa de que los convenios contractuales con los constructores de los embalses establecen que debe existir cooperación con las comunidades locales, es posible asumir que desde el principio se reconoció lo delicado del asunto. Resulta claro que algunas protestas tuvieron lugar desde el inicio del proceso y que hubo indignación sobre ciertas declaraciones engañosas de las autoridades relativas a la naturaleza de los embalses, como, por ejemplo, que los contratos tenían una duración de cinco años, cuando en realidad su extensión era de 25 años.

El aspecto más evidente que debe señalarse, como hace hincapié Diepart, es que la planicie de inundación desde tiempo inmemorial ha sido una fuente de recursos de uso común para la pesca, el pastoreo del ganado y la recolección de productos no madereros. La concesión de tierras produce la pérdida del acceso tradicional a ellas y se convierte en lo que, en términos marxistas clásicos, podría describirse como una acumulación primitiva. ${ }^{24}$ Diepart arguye que el valor de los recursos que se han perdido equivale al valor generado por los campos de arroz irrigados de la estación seca.

El primer informe publicado que encontramos sobre las disputas relacionadas con los embalses de irrigación es un estudio, de 2006, elaborado por el Centro de Estudios Avanzados de Camboya, y dedicado a las disputas de tierras y a la forma como estas disputas se resuelven en Camboya en el contexto de la nueva ley relativa a la propiedad de la tierra y de las formas recientemente descentralizadas del gobierno local. Uno de

${ }^{24}$ Es cierto que, en general, las concesiones de tierras más recientes estipulan que se mantendrán las formas tradicionales de acceso a los recursos, como, por ejemplo, los estanques de peces. 
los tres casos que examina el informe se refiere a disputas por tierras en 2005 en el distrito de Stoung, en Kampong Thom. Allí se revisa la historia de tres aldeas donde tuvieron lugar disputas sobre la construcción de embalses para irrigación que había aprobado el jefe del subdistrito. No se da el nombre del subdistrito, pero aparentemente es uno colindante con Samprouch. En dos de las tres aldeas, la población local opuso una resistencia importante. En una (Aldea A), los aldeanos se habían enterado del proyecto mientras aún lo estaba negociando la comunidad del subdistrito:

A pesar de que los habitantes de la aldea no estaban de acuerdo con el proyecto, la autoridad provincial otorgó al inversionista la aprobación del proyecto, y éste empezó a llevar su equipo de construcción al lugar. Esto provocó que los pobladores de la aldea fueran al campo, armados con cuchillos y hachas, para impedir la ocupación de sus tierras, y que amenazaran con incendiar el equipo si se procedía a la construcción. El jefe de la aldea y un consejero de la comunidad se presentaron inmediatamente en la escena y pidieron a los enfurecidos aldeanos que no usaran la violencia y que resolvieran en forma pacífica la disputa. Ambos bandos acordaron parar la construcción provisionalmente hasta que quedara resuelta la disputa entre los pobladores de la aldea y el inversionista. ${ }^{25}$

En este proceso se involucraron representantes del partido de oposición y de organizaciones de derechos humanos. A causa de las protestas y de las reuniones, el gobernador provincial decidió " parar la realización del proyecto de inversión y permitir a los aldeanos usar las tierras en disputa con la condición de que ese año cultivaran arroz". ${ }^{26}$ Finalmente, el inversionista decidió abandonar el proyecto de construcción de un embalse. Los acontecimientos que ocurrieron en otra aldea (Aldea B) tuvieron un desarrollo análogo: una confrontación con los aldeanos armados, a la cual siguieron reuniones y una decisión similar del gobernador provincial, con la única diferencia de que, cuando se escribió el informe, el inversionista no había abandonado sus intenciones. Una tercera aldea (Aldea C) decidió que, en lugar de protestar, ejercería presión para que el inversionista les entregara una compensación financiera, lo cual

${ }^{25}$ Center for Advanced Study, "Justice for the Poor?”, op. cit., pp. 52-53.

${ }^{26}$ Ibid., p. 54. 
pudo negociarse con éxito, aunque algunos aldeanos aceptaron a regañadientes, pensando que no les quedaba otra opción.

Es difícil hacer generalizaciones acerca de la relación entre los proyectos de embalses para irrigación y las poblaciones rurales adyacentes. Es incuestionable que ha habido conflictos significativos, y en nuestro análisis esto está vinculado a algo básico: al cambio subyacente de la relación con los recursos. Pero al tratar de entender lo que se podía dilucidar más claramente sobre los conflictos en la planicie de inundación, pudimos apreciar que algunos embalses eran aparentemente causa de mayores conflictos que otros.

Se han celebrado diversos tipos de arreglos en diferentes contextos. En algunos de los casos más tempranos, los inversionistas, al mismo tiempo que construían dos embalses para ellos, construían un tercero para que lo usaran los aldeanos. Esto, según lo describen Ngo y Chan, ocurrió en el subdistrito de Chamnar Kraom. (El inversionista Iev Vanna nos dijo que había hecho esto en el subdistrito de Kampong Ko). Los embalses de este tipo que describen Ngo y Chan son administrados por funcionarios del subdistrito, y a los aldeanos más pobres se les $\mathrm{da}$ acceso a la tierra irrigada año con año en forma rotativa. Ngo y Chan afirman que éste es el arreglo que más "favorece a los pobres", en los diferentes tipos de embalses. Esto puede representar que los inversionistas procuren atenerse al arreglo contractual para trabajar $40 \%$ de la tierra irrigada en forma conjunta con los pobladores de las aldeas. Desgraciadamente, esta práctica no ha continuado, tal vez porque los inversionistas se dieron cuenta de que no estaban obligados a cumplir el requerimiento de $40 \%$; en el subdistrito de Samprouch no existen embalses sometidos a este arreglo.

La evidencia sobre el grado en el que los embalses han generado tasas de empleo significativas para los aldeanos es algo contradictoria. Ngo y Chan, en su estudio de los subdistritos de Chamnar Kraom y de Samprouch, sostienen que sí se han creado trabajos en forma importante, lo cual constituye un argumento en favor de los embalses. Diepart, en su trabajo de 2010 sobre el subdistrito de Srayov, en Kampong Thom, sostiene las conclusiones de Ngo y Chan, en tanto que sobre los subdistritos de Samprouch y Msa Krong, en 2007, escribió: 
La mayor parte de los habitantes locales entrevistados del área (los subdistritos de Msa Krong y Samprouch) dijeron que hasta ese momento no habían sido reclutados para la construcción de los embalses ni tampoco como trabajadores asalariados en las áreas cultivadas. Los empresarios investigados afirmaron que esto se debía a que querían asegurarse de que el sistema podía funcionar sin el involucramiento de los campesinos locales durante los primeros años. ${ }^{27}$

Nuestras propias investigaciones, basadas en muestreos menos científicos, tendieron a apoyar la afirmación de Diepart de 2007. Se nos dijo que, a causa de una mayor mecanización de los embalses empresariales, se requería menos mano de obra y que, además, no todo el trabajo requerido provenía de la población general del subdistrito. Los inversionistas podían favorecer a sus propios parientes o traer trabajadores de Siem Reap.

Algunos propietarios, en tanto que mantienen el control de sus embalses, venden a agricultores locales parte de la tierra que dichos embalses alimentan; otros rentan la tierra, y en unos cuantos casos se ha trabajado la tierra bajo el régimen de aparcería. Además de acuerdos más formales con tierras que quedan inmediatamente abajo del embalse que las alimenta, hay arreglos cada vez menos formales con campesinos que usan el agua que sigue fluyendo más allá de estas tierras. Algunos dueños de los embalses permiten que los agricultores usen el agua en forma gratuita; otros les cobran cuotas simbólicas.

En Samprouch hay dos embalses vinculados a aldeas específicas. En un caso, la aldea posee y opera el embalse; en otro, la aldea concede su derecho sobre el embalse a un empresario. En un subdistrito adjunto, en la década de 1980, la tierra que actualmente se encuentra ocupada por un embalse se asignó para el cultivo de arroz flotante a otro subdistrito situado allende de la carretera nacional. Esta otra comunidad hoy posee el embalse del subdistrito más cercano al Tonlé Sap en forma oficial, pero renta su derecho a la tierra a un empresario dedicado a la irrigación; se trata de un acuerdo legal que, no obstante, resulta conflictivo para los residentes del subdistrito donde está situado el embalse. 
Mientras que en muchos lugares los aldeanos se han opuesto a que empresarios construyan embalses para riego, o han considerado que no se les ha compensado adecuadamente por haber perdido el acceso a la tierra, hay campesinos que, habiendo concertado acuerdos con uno de los embalses empresariales para el uso agrícola del agua, dependen de este acuerdo para su sustento, y ahora se encuentran vulnerables si se aplica una política destinada a destruir los embalses.

Todo esto nos conduce al caso del "embalse del pueblo", en el subdistrito de Samprouch, que constituye el tema del presente artículo. Empezaremos señalando que, a pesar de la posible connotación que la frase "embalse del pueblo" pueda tener, no es la historia de un proyecto izquierdista o activista (aunque haya tenido su origen en las protestas); quisiéramos describirlo como el resultado de procesos de negociación complejos, los cuales estuvieron manchados por acusaciones de corrupción y de complicidad con las autoridades, además de haber sido construido en áreas cuestionables desde el punto de vista ambiental; pero que, sin embargo, representó una solución práctica, por lo menos para algunos de los aldeanos.

La modalidad de la disputa en el subdistrito de Samprouch coincide con algunos aspectos que se describen en el estudio del Centro de Estudios Avanzados sobre otras partes de la provincia. Aunque el embalse del pueblo ya se estaba constituyendo cuando realizamos las investigaciones preliminares en el subdistrito, en julio de 2009, lo más relevante en nuestras conversaciones con los aldeanos fue el sentimiento de haber sufrido agravios y su intención de organizarse a causa de ellos. Tales agravios eran de diferentes tipos (y algunos pueden haber sido más importantes que otros en su momento): había quejas de que se les había hecho creer que los contratos para los embalses tenían una duración de cinco años, cuando en realidad se prolongaban por 25 años; o que simplemente se les había incitado a poner sus huellas digitales en algunos documentos sin que se les hubieran explicado las implicaciones que ello conllevaba. Algunos aldeanos se quedaron con la impresión de que los embalses pasarían a los propios aldeanos después de cierto número de años, pero eso no estaba ocurriendo. Hubo informes de que el jefe del subdistrito había animado a la gente 
a "vender" la tierra, a pesar de que la compensación era mínima (y había temor de que, si no aceptaban ese dinero, no recibirían ninguna compensación). Hubo discusiones entre ministerios que apoyaban a las diferentes partes en disputa. La gente había acudido a la organización no gubernamental denominada Liga Camboyana para la Promoción y la Defensa de los Derechos Humanos y a otras organizaciones no gubernamentales en la capital de la provincia para solicitar su ayuda. Uno de los dueños de los embalses, C., contaba con policía armada que amenazaba a la gente.

También hubo lo que ahora entendemos como quejas de agricultores individuales a los que se les había otorgado previamente acceso a la tierra de la planicie de inundación con propósitos específicos y cuyos derechos quedaron anulados por los embalses construidos recientemente. Un hombre que recurrió a la capital provincial tuvo éxito limitado; limitado porque, a pesar de que contaba con una autorización para el uso de la tierra que le había otorgado el subdistrito con anterioridad a la concesión de tierra al inversionista del embalse, nunca había contado con una autorización provincial. Sin embargo, este hombre quedó satisfecho con el resultado, pues otras personas - según dijo- habían perdido toda su tierra porque no se habían atrevido a pelear sus derechos en la capital provincial.

La insatisfacción alcanzó su punto crítico en las elecciones subdistritales de 2007, cuando un candidato del partido opositor, el Partido Sam Rainsy (PSR), fue elegido frente al candidato titular, perteneciente al dominante Partido del Pueblo de Camboya (PPC). ${ }^{28}$ Por lo pronto, la elección de un jefe del subdistrito perteneciente al PSR le indicaba a la oficina del gobernador provincial que había problemas significativos relacionados con los embalses que debían ser resueltos.

Uno de los líderes de las manifestaciones contra los embalses era un agricultor de alrededor de 40 años a quien denominaremos P. P. En ese tiempo, P. P. y otros manifestantes

${ }^{28}$ El candidato del PSR era hermano de su oponente, el titular del consejo del subdistrito. Las implicaciones políticas de este hecho no son claras, dado que el poder del jefe del consejo ha estado circunscrito en forma significativa al PPC, y este partido decide la mayor parte de los asuntos; sin embargo, el nuevo jefe del consejo del subdistrito ha apoyado el embalse del pueblo. 
decidieron que, en vez de protestar contra los embalses existentes, debían presionar para obtener el control de un embalse. En nuestras conversaciones con los aldeanos generalmente se identificaba a P. P. como el líder del embalse del pueblo, aunque después supimos que dicho embalse era administrado por un comité integrado por tres personas.

Según relataron, se entregó al subdistrito un lote grande de terreno (360 hectáreas) de la planicie de inundación situado en un nivel de embalses bastante bajo; tierra que ya se le había prometido a otro inversionista, a C., pero éste se había demorado en construir el embalse porque al mismo tiempo construía otro de mayores dimensiones. Frente a las protestas contra C., el gobernador de la provincia, Nam Tum, entregó la tierra al "pueblo" del subdistrito en marzo de 2006. Parte de la justificación para hacer esto fue que C. se había demorado más de dos años en construir el embalse desde que se le había otorgado el permiso para hacerlo. Representantes del gobierno provincial asistieron a reuniones celebradas con los aldeanos en uno de los wat; los participantes afirmaron que se había formado una "comunidad" con el reconocimiento de las autoridades.

En la primera mitad de 2010, durante nuestro trabajo de campo, surgieron diversos problemas, y las tensiones todavía eran muy evidentes. P. P. es pariente de dos hermanos que son el jefe y el ex jefe del subdistrito. En realidad, no hay gran cosa que lo distinga de otros aldeanos, pero su hermano es maestro de escuela en la aldea, y él cuenta con una educación ligeramente superior a la de la mayor parte de sus vecinos..$^{29} \mathrm{Su}$ familia tradicionalmente ha estado involucrada en el comercio de pescado, y su modesta casa de madera debe ser un poco mayor que las casas de su alrededor. Llegó a involucrarse en el embalse del pueblo a causa de su participación en protestas anteriores. Fue electo en reuniones de la comunidad para que los representara en las pláticas con C. y con otro propietario de un embalse grande, $\mathrm{K}$. Aunque durante ese tiempo recibió amenazas violentas, no hay indicaciones de que sea un activista en el sentido de que esté ideológicamente motivado. Su involucramiento en el embalse

${ }^{29}$ También es primo de un monje muy conocido que actualmente es el abad de un wat de Phnom Penh. 
lo puso en contacto con el que a la sazón era gobernador de la provincia y que actualmente ya dejó su cargo, Nam Tum, a quien menciona en las conversaciones. Él mismo tiene un pequeño embalse de 60 hectáreas que, aunque fue construido después del embalse del pueblo, se encuentra, según dice, en un terreno que él adquirió antes de la construcción del embalse del pueblo. De este modo, aparte del embalse del pueblo, tiene inversiones en la economía de los embalses. ${ }^{30}$

Aunque Nam Tum y otros representantes del gobierno provincial estuvieron presentes en las juntas para crear el embalse, y pueden haber ayudado a concebir el plan para su creación, en ningún sentido está claro que exista una base legal para el embalse del pueblo. Mientras que en el caso de los embalses de empresarios se firmaron contratos, el jefe del subdistrito nos dijo que, en el caso del embalse del pueblo no se había hecho esto porque "no era necesario". El jefe provincial del Ministerio de Agricultura, Silvicultura y Pesca para Kampong Thom nos dijo que la comunidad del embalse había solicitado reconocimiento formal como una cooperativa agrícola, pero que él había rechazado la solicitud porque la tierra de cultivo alimentada por las aguas del embalse se extendía claramente a áreas de manglares y porque, además, tenían dudas acerca de P. P., quien era propietario de otro embalse, por lo que podía estar vinculado a intereses externos. El Ministerio de Recursos Hídricos y Mineralogía tampoco reconoce el embalse del pueblo como un grupo agrícola de usuarios de agua (no hay comunidades agrícolas de usuarios de agua registradas formalmente en la planicie de inundación del Tonlé Sap en Kampong Thom).

Tras la integración como una comunidad, los arrozales que quedaban bajo el embalse del pueblo fueron asignados por sorteo a las familias participantes: a 1009 familias se les asignó una hectárea. Durante nuestra investigación, de 2009 y 2010, los miembros de estas familias trabajaban arduamente desmontando las áreas de manglares para usarlas como arrozales alimentados con agua del embalse. Este tipo de trabajo fue descrito unánimemente como extremadamente duro, agotador. Ellos sa-

${ }^{30}$ Un argumento que se esboza para desacreditarlo es que usó contribuciones de la gente destinadas al embalse del pueblo para comprar su propio embalse. 
bían que era ilegal y que se les podía imponer una multa equivalente a 200 dólares estadounidenses si eran descubiertos. Sabían que con esto se destruía el lugar de desove de los peces y se sentían avergonzados por ello; se reían con bochorno mientras bebían vino de palma, pero consideraban que no les quedaba otra opción si querían sobrevivir como agricultores. (Hasta hace poco, los aldeanos complementaban su ingreso en forma significativa con la pesca en la planicie de inundación. En un círculo vicioso, ahora hay menos peces - tal vez a causa de los embalses-, por lo que se ven obligados a derrumbar más manglares, situación que, a su vez, contribuirá a que disminuyan más los peces).

La queja principal contra el embalse del pueblo es que, a pesar de que tiene el propósito de ser para todos los residentes del subdistrito, muchos de ellos no han podido participar. De las quince aldeas existentes en el subdistrito, sólo doce participan en el embalse y, de estas doce, algunas lo hacen con más personas que otras. Posiblemente la causa de que algunas personas no participen se deba a que no se unieron al proyecto con suficiente anticipación; algunos otros creyeron que no podrían pagar la cuota equivalente a 200 dólares estadounidenses que se les cobraba, lo cual, en cierta medida, puede representar una discriminación hacia los más pobres de los pobres. La ira llegó a tal extremo que, en 2008, un grupo de agricultores del subdistrito fue a Phnom Penh a protestar en la Asamblea Nacional. (Dado que C. aún se esfuerza por recuperar los derechos de propiedad del embalse, ha habido acusaciones en el sentido de que apoyaba a las personas que protestaban. Algunas personas con las que nos entrevistamos dijeron que esto era falso, aunque otros aldeanos pueden haber creído sinceramente que los acuerdos para el trabajo agrícola en forma cooperativa en el embalse de C. serían más benéficos). ${ }^{31}$ Aunque las protestas en Phnom Penh no recibieron apoyo alguno del gobierno nacional, Nam Tum pidió que se abriera el embalse a todos los aldeanos del subdistrito. P. P. dijo que aceptaría contribuciones menores de otros aldeanos que formaran parte de un nuevo embalse cuando

${ }^{31}$ Un grupo de 800 familias apoyaron a C, quien ofrecía un arreglo mediante el cual los agricultores podían trabajar la tierra alimentada por su embalse a cambio de un pago de entre 800 y 1000 rieles por cada hectárea trabajada. 
encontrara el terreno para hacerlo; pero no podía garantizar que lo iba a encontrar o que regresaría el dinero si no se llegaba a construir el embalse. Esto le valió críticas de que tomaba dinero para su propio beneficio. También ha habido críticas de los miembros del embalse porque consideran que el dinero se ha pedido en forma arbitraria - por ejemplo, una cuota para tener derecho a desmontar lotes de terreno- y que ha ido a parar a los bolsillos de los administradores y no al proyecto del embalse. No citamos estos relatos para atacar a P. P. o al embalse del pueblo - por lo que hemos oído hasta ahora, creemos que la mayor parte de las acciones de P. P. estaban justificadas-, sino para transmitir la idea de que el proyecto del embalse aún está caracterizado por dudas y, como los dolores de parto, por muchos aspectos aún sin resolver. A su favor está el hecho de que une a los participantes de niveles marginales de subsistencia con el objetivo de negociar su sustento cotidiano.

Durante varias visitas a Samprouch, en la primera mitad de 2010, nos encontramos con aldeanos que estaban enterados de que se estaban destruyendo algunos embalses, pero esto no les preocupaba en exceso y parecían optimistas acerca de que su embalse constituiría una excepción. En julio, en nuestra última visita al subdistrito, sin embargo, encontramos que muchos aldeanos afiliados al embalse habían permanecido en el lugar del embalse durante los últimos tres días debido al hecho de que varios embalses del subdistrito colindante, Msa Krong, habían sido desmantelados en poco tiempo por equipos trasladados en helicóptero, de tal forma que tenían miedo de que las autoridades se dirigieran posteriormente a Samprouch. Varios miembros del embalse del pueblo habían acampado durante varios días en el lugar, preparados para defenderlo si había intentos de destruirlo. P. P. posteriormente nos diría que todos, los 1009 miembros del embalse, habían permanecido allí. (Aunque, sin duda, los miembros de la comunidad del embalse estaban deseosos de participar, después oímos algunas narraciones de que P. P. les había dicho que cobraría una multa a los que no asistieran al lugar en aquel momento). Cuando llegamos, el asunto se había resuelto en su mayor parte, pero aún podían verse los restos de los cobertizos improvisados donde habían estado durmiendo los aldeanos. Alrededor del embalse 
habían colocado palos con pequeñas banderas camboyanas, y debajo de ellas habían puesto banderas con el símbolo del PPC, el partido dominante (lo que resultaba llamativo, ya que la aldea había votado por el PSR, el partido opositor, en las elecciones subdistritales). Representantes del embalse nos dijeron (al igual que P. P. cuando nos entrevistamos con él posteriormente) que no estaban protestando sino pidiendo misericordia a las autoridades para que no destruyeran el embalse. Un helicóptero que transportaba al ministro de Recursos Hídricos y Mineralogía había sobrevolado el lugar para inspeccionarlo. También habían ido periodistas de Phnom Penh (pero cuando llegamos ya no estaban allí). Un grupo de representantes de los ministerios involucrados los había visitado y les había dado postes. Se les dijo que tenían que ser muy cuidadosos en demarcar los límites del terreno del embalse del pueblo, lo que parecía indicar que éste no sería demolido cuando finalmente se emprendiera la destrucción de los otros embalses. Esto también significaba que no debía haber más desmonte de terreno fuera de los límites que se habían establecido. El 6 de julio se envió una carta formal para solicitar que se permitiera la existencia del embalse, la cual había sido sellada de recibida por el jefe del subdistrito, por el jefe del distrito y por el presidente del comité provincial, y supuestamente se le haría llegar a Hun Sen. Posteriormente, un funcionario provincial nos confirmó que el embalse no sería destruido.

A algunos aldeanos se les asignó la responsabilidad de monitorear con regularidad los postes de los bordes. Por conversaciones con ellos, tuvimos la impresión de que, a pesar de que no recibían paga alguna, se tomaban esta tarea con seriedad.

En una visita, P. P., con cierta arrogancia, dijo a Chhuon Hoeur que él era la única persona cualificada para ser jefe del subdistrito. Se presentó como candidato del PPC para contender por el puesto en las elecciones locales de 2012 y ganó. No obstante, algunos sectores del subdistrito han criticado constantemente a P. P. En una breve visita que realizamos en 2012, poco después de su elección, eran numerosas las críticas en el sentido de que se había pasado de la raya. Se contaba que estaba involucrado con un grupo de empresarios que estaban desmontando áreas ilegalmente en la zona de manglares, dentro 
del área claramente demarcada como protegida (tal involucramiento puede haber consistido solamente en el uso de su equipo en dicha tarea). Lo buscamos el día anterior a nuestra partida, pero fue imposible encontrarlo. Su familia parecía estar sinceramente desconcertada por no poder contactarlo por teléfono. Al día siguiente, cuando nos encontrábamos en el autobús que nos llevaría a Phnom Penh, nos enteramos de que había sido arrestado, junto con otras dos personas, por sus vínculos con el desmonte ilegal de los manglares. De las otras dos personas arrestadas, una era el jefe provincial de Kampong Thom, perteneciente al Ministerio de Recursos Hídricos y Mineralogía (a quien también habíamos conocido y con quien nos habíamos entrevistado). Las noticias de los arrestos se transmitieron en la televisión nacional y se publicaron en los periódicos. En estos reportes se mencionaba que habría más arrestos; sin embargo, esto nunca sucedió, lo que sugiere que los tres arrestados eran chivos expiatorios, o tal vez que sus arrestos tenían la intención de intimidar a otras personas con el fin de que no intentaran evitar el arresto mediante sobornos. De acuerdo con sus parientes, P. P. durante ese tiempo había contado con el apoyo de Nam Tum, a la sazón jefe del consejo de Kampong Thom, aunque tal apoyo no fue suficiente para evitar su encarcelamiento durante un año. A lo largo de ese año, el anterior jefe del subdistrito (representante de un partido opositor) asumió los deberes de P. P.

Tras un año de prisión, a P. P. lo recibió en la puerta de salida de la prisión un abogado de Nam Tum que lo condujo en automóvil a uno de los mayores templos budistas de Phnom Penh. Allí participó en una ceremonia compleja de bendición con agua destinada a liberarlo de cualquier fuerza negativa que pudiera haber acumulado durante su periodo de arresto y también para elevarlo a un nivel espiritual de poder más intenso. A pesar de que, de acuerdo con las leyes camboyanas, alguien con un historial delictivo no puede ocupar un cargo político, se le permitió volver a ocupar su puesto como jefe del subdistrito.

No mucho después de que sucediera esto realizamos una breve visita al subdistrito y encontramos que los aldeanos no estaban dispuestos a hablar de P. P. En las elecciones nacionales, 
que se celebraron poco después, a finales de julio de 2013 (que no implicaban votaciones para elegir funcionarios locales), el subdistrito votó por el Partido Nacional del Rescate de Camboya, creado recientemente por la unión de dos partidos opositores.

Mientras que el arresto de P. P. indica la determinación del gobierno de no permitir embalses en las áreas recientemente protegidas y demarcadas en las márgenes del lago Tonlé Sap, los embalses que ya existen, entre ellos el embalse del pueblo, ya no se cuestionan y no parecen estar en riesgo.

\section{Conclusiones}

¿Qué significa todo esto? Desde nuestro punto de vista está relacionado con un cambio importante del régimen de la tierra que tiene que ver no sólo con las nuevas leyes relativas a la propiedad de la tierra, sino también con el cambio de las relaciones económicas y con el cambio de las relaciones del Estado y de otros actores con los recursos naturales. Más arriba citamos a Bloch, quien afirma que toda la obra de Marx y Engels "prueba que la propiedad es representada por la ideología como una relación entre la gente y las cosas, pero que en términos materiales se trata de una relación social". ${ }^{32}$ A pesar de las reservas formuladas, consideramos que tal aseveración es, en gran medida, cierta (incluso sin considerar que significa que un régimen de la tierra determinado inevitablemente sugiere ciertas relaciones sociales dadas). Evidentemente, el cambio llama la atención hacia el hecho de que las relaciones de "propiedad" puedan ser ambiguas y sujetas a negociación. La situación particular que examinamos tiene lugar en un marco especialmente vulnerable en términos ambientales, en un momento en que se está dando un cambio ambiental, el cual se convierte en parte de la dinámica y contribuye a aumentar la complejidad moral de la situación.

Lo que describimos es una adaptación histórica al cambio que, hasta cierto punto, podría denominarse "resistencia”, pero

${ }^{32}$ Bloch, "La propiedad y el final de la alianza”, op. cit., p. 204. 
no en el sentido de una lucha unificada y militante, sino en un sentido más fluido de negociación, readaptación y, en algunos casos, compromiso. Apreciamos ciertos aspectos de derrota, pero también vemos la movilización de las comunidades rurales en lo que parecen ser nuevas rutas.

Sherry Ortner escribe:

Los estudios sobre la resistencia son débiles porque son etnográficamente débiles: débiles en lo que se refiere a las políticas internas de los grupos dominados; débiles en lo que se refiere a la riqueza cultural de esos grupos; débiles en lo que se refiere a la subjetividad -las intenciones, los deseos, los temores, los proyectos- de los actores involucrados en estos dramas. ${ }^{33}$

Esperamos que nuestro estudio al menos dé sentido a parte de la complejidad y de la ironía de la "resistencia".

Desde un punto de vista estrictamente ambiental, el embalse del pueblo constituyó un proyecto negativo en cuanto que implicaba mayor intrusión en el área de los manglares de la planicie de inundación del Tonlé Sap. En términos políticos, en cierto modo puede haber representado una derrota, hasta el punto de que la población de un subdistrito que había votado por un partido de oposición acabó colocando banderas del partido dominante, y le pedía misericordia. También puede afirmarse que el embalse del pueblo estaba entonces lejos de haber tenido éxito por estar plagado de disputas internas. No obstante, encontramos algo positivo en el hecho mismo de que agricultores del arroz pudieran unirse para crear una alternativa de trabajo frente a los embalses de propiedad privada que proliferaban a su alrededor, y que aparentemente podrían haberles quitado su medio de subsistencia.

El embalse del pueblo no tuvo la clase de apoyo estatal que, en años recientes, se ha dado a la administración de recursos de base comunitaria, o a grupos agrícolas de usuarios de agua o cooperativas agrícolas. No lograron obtener el tipo de reconocimiento oficial con el que cuentan estos grupos. No obstante, creemos que el espíritu de la época de estas otras

${ }^{33}$ Citado en Tania Murray Li, The Will to Improve: Governmentality, Development, and the Practice of Politics, Durham, Duke University Press, 2007, p. 157. 
clases de organizaciones rurales puede tener cierta relación con el movimiento creado alrededor del embalse del pueblo. La comunidad se unió inicialmente motivada por las protestas y, posteriormente, por el deseo común de crearse una oportunidad en una situación difícil.

\section{Traducción del inglés: Gabriela Uranga}

Dirección institucional de los autores:

John Marston

Centro de Estudios de Asia y África

El Colegio de México

Camino al Ajusco 20

Pedregal de Sta. Teresa

10740, México, D.F.

jmars@colmex.mx

Chbuon Hoeur

Trav Phny Village

Tang Krasang Commune

Batheay District

Kampong Cham

hoeur@hotmail.com

\section{Bibliografía}

Banco Mundial, Cambodia: Halving Poverty by 2014? Poverty Assessment 2006, Informe núm. 35213-KH, Nueva York, Banco Mundial.

BLOCH, Maurice, "La propiedad y el final de la alianza", en M. Bloch (comp.), Análisis marxistas y antropología social, Barcelona, Anagrama, 1977, pp. 241-268.

Cambodian Development Resource Institute, Empirical Evidence of Irrigation Management in the Tonle Sap Basin: Issues and Challenges, documento de trabajo núm. 48, Phnom Penh, CDRI, 2010.

Cambodian Office of the High Commissioner for Human Rights, "Economic Land Concessions in Cambodia: A Human Rights Perspective", Phnom Penh, OHCHR, 2007. 
Center for Advanced Study, "Justice for the Poor? An Exploratory Study of Collective Grievances and Land and Local Governance in Cambodia”, Phnom Penh, Center for Advanced Study-Banco Mundial, 2006.

CHem, Phalla et al., Framing Research on Water Resources Management and Governance in Cambodia: A Literature Revierw, Phnom Penh, Cambodia Development Resource Institute, 2008.

CHou, Chea, The Local Governance of Commonpool Resources: The Case of Irrigation Water in Cambodia, Phnom Penh, Cambodia Development Resource Institute, 2010.

Department of Planning and Statistics, Agriculture Census of Cambodia, Phnom Penh, Ministry of Agriculture, Forestry and Fisheries, 18-21 de febrero de 2014.

Diepart, Jean-Christophe, "Cambodian Peasant's Contribution to Rural Development: A Perspective from Kampong Thom Province", Biotechnology, Agronomy, Society and Environment, vol. 14, núm. 2, 2010, pp. 321-340.

Diepart, Jean-Christophe, "Problèmes et enjeux de l'économie rurale au Cambodge: Entre nouvelles gouvernances et réalités paysannes: Le cas de la province de Kampong Thom”, tesis de doctorado, Académie Universitaire Wallonie-Bruxelles-Faculté Universitaire des Science Agronomiques de Gembloux, Communauté française de Belgique, 2007.

DiePart, Jean-Christophe, "Recent Land Dynamics in the Tonle Sap Flood Plain and its Impact on the Local Communities", Tonle Sap Biosphere Reserve Bulletin, vol. 3, 2007, pp. 20-22.

Fox, Jeff y Judy Ledgerwood, "Dry-Season Flood-Recession Rice in the Mekong Delta: Two Thousand Years of Sustainable Agriculture?", Asian Perspectives, vol. 38, núm. 1, 1999, pp. 3750 .

Gray, Thomas N. E., Nigel J. Collar, Peter J. A. Davidson et al., "Distribution, Status, and Conservation of the Bengal Florican Houbaropsis Bengalensis in Cambodia", Bird Conservation International, vol. 19, núm. 1, 2009, pp. 1-14.

Harvey, David, The New Imperialism, Nueva York, Oxford University Press, 2003.

KHIEv, Daravy, "Challenge of Participant Irrigation Management in Cambodia: The Case of Damnak Ampil Irrigation Scheme", tesis de maestría, Universidad de Sidney, Sidney, 2010.

Kноuтн, Sophakchakrya, "Progress Made on Illegal Reservoirs", Phnom Penh Post, 9 de marzo de 2011.

LANDO, Richard P. y Solieng Mak, Deepwater Rice in Cambodia: A 
Baseline Survey, Manila, International Rice Research Institute, 1994.

LI, Tania Murray, The Will to Improve: Governmentality, Development, and the Practice of Politics, Durham, Duke University Press, 2007.

Marston, John A. (coord.), Antropología y comunidad en Camboya: Reflexiones sobre la obra de May Ebihara, México, El Colegio de México, 2011.

Middleton, Carl y Tola Prom, "Community Organization for Managing Water Resources around Tonle Sap Lake: A Myth or a Reality”, en Matti Kummu, Marko Keskinen y Olli Varis (comps.), Modern Myths of the Mekong, Helsinki, Universidad Tecnológica de Helsinki, 2008, pp. 149-159.

MuUkKonen, Satu, "Water Management in Cambodia-Resources and Relations", tesis de maestría, Universidad de Helsinki, Helsinki, 2007.

National Institute of Statistics, General Population Census of Cambodia 2008: Provisional Population Totals, Phnom Penh, National Institute of Statistics, 2008.

NGo, Sothath y Chan Sophal, Does Large Scale Agricultural Investment Benefit the Poor?, Phnom Penh, Cambodian Economic Association, junio de 2010.

ÖJENDAL, Joakim, "Sharing the Good: Modes of Managing Water Resources in the Lower Mekong River Basin", tesis de doctorado, Universidad de Gotemburgo, Gotemburgo, 2000.

Ostrom, Elinor, Governing the Commons: The Evolution of Institutions for Collective Action, Cambridge-Nueva York, Cambridge University Press, 1990.

Ros, Bandeth, "Participatory Irrigation Management and Factors that Influence the Success of Farmer-Water User Communities: A Case Study in Cambodia”, tesis de maestría, Universidad de Massey, Auckland, 2010.

SAK, R., Human Territoriality: Its Theory and History, Cambridge, Cambridge University Press, 1986.

SiтHIRITH, Mak, Cooperation in the Mekong River Basin: A Reflection of Cambodia's Experiences in the Development of the Mekong Region, Chiang Mai, Regional Center for Social Science and Sustainable Development, 2007.

Somatra, Kim Sean, "Territoriality and State Power in Cambodia: The Case of Demarcation in Tonle Sap", tesis de doctorado, Universidad de Sidney, Sidney, 2013.

Someth, P., N. Kubo, H. Tanji y S. Ly, "Ring Dike System to Harness 
Floodwater from the Mekong River for Paddy Rice Cultivation in the Tonle Sap Lake Floodplain in Cambodia", Agricultural Water Management, vol. 96, núm. 1, 2009, pp. 100-110.

Un, Kheang y Sokbunthoeun So, "Land Rights in Cambodia: How Neopatrimonial Politics Restricts Land Policy Reform", Pacific Affairs, vol. 84, núm. 2, 2011, pp. 289-308.

VAnDERgeEst, Peter y Nancy Lee Peluso, "Territorialization and State Power in Thailand", Theory and Society, vol. 24, núm. 3, 1995, pp. 385-426. 